\title{
AN UPDATE ON FLUID BIOMARKERS FOR NEURODEGENERATIVE DISEASES: RECENT SUCCESS AND CHALLENGES AHEAD.
}

\section{AUTHORS}

Joel Simrén ${ }^{1}$, Nicholas J. Ashton ${ }^{1,2,3,4}$, Kaj Blennow ${ }^{1,5}$, Henrik Zetterberg ${ }^{1,5,6,7}$

\section{AFFILIATIONS}

${ }^{1}$ Department of Psychiatry and Neurochemistry, Institute of Neuroscience \& Physiology, the Sahlgrenska Academy at the University of Gothenburg, Mölndal, Sweden; ${ }^{2}$ Wallenberg Centre for Molecular and Translational Medicine, University of Gothenburg, Gothenburg, Sweden; ${ }^{3}$ King's College London, Institute of Psychiatry, Psychology and Neuroscience, Maurice Wohl Institute Clinical Neuroscience Institute, London, UK; ${ }^{4}$ NIHR Biomedical Research Centre for Mental Health and Biomedical Research Unit for Dementia at South London and Maudsley NHS Foundation, London, UK; ${ }^{5}$ Clinical Neurochemistry Laboratory, Sahlgrenska University Hospital, Mölndal, Sweden; ${ }^{6}$ Department of Neurodegenerative Disease, UCL Institute of Neurology, Queen Square, London, UK; ${ }^{7}$ UK Dementia Research Institute at UCL, London, UK.

\section{CORRESPONDING AUTHOR}

Henrik Zetterberg, MD, PhD, Department of Psychiatry and Neurochemistry, Institute of Neuroscience and Physiology, The Sahlgrenska Academy, University of Gothenburg, S-431, 80 Mölndal, Sweden (henrik.zetterberg@gu.se) 


\section{ABSTRACT}

Over the last twenty years, the characterization of Alzheimer's disease (AD) patients has progressed from a description of clinical symptomatology followed by neuropathological findings at autopsy to detailed in vivo pathophysiological signatures using cerebrospinal fluid (CSF) and positron emission tomography (PET). Additionally, CSF biomarkers now also reflect synaptic pathology, axonal injury and neuroinflammation. Technological advances have provided ultrasensitive techniques capable of measuring proteins of pathophysiological importance at femtomolar concentrations in blood samples (e.g. amyloid, tau species and neurofilaments). This has huge potential to screen large populations in the near future, which is essential for secondary prevention trials and primary care management. For most neurodegenerative diseases, however, research has not reached the same success. Common pathologies, such as that underlying dementia with Lewy bodies, Parkinson's disease and frontotemporal dementias, are still without reliable diagnostic biomarkers, although emerging techniques show promising pilot results for some of these diseases. This is likely to change in the next few years, which will be of great importance to stratify populations enrolling in clinical trials, given that these pathologies often coexist.

\section{INTRODUCTION}

Neurodegenerative diseases (NDD) comprise a group of neurological conditions that share the trait of nerve cell death, together with signature protein inclusions found in autopsies, which is why they are sometimes referred to as proteinopathies. NDDs include a large number of diseases, but most can be classified by the presence of amyloid-beta (A $\beta)$, tau, $\alpha$-synuclein, prion protein (PrP), and transactive response DNA-binding protein 43 (TDP-43), resulting in cerebral amyloidosis, tauopathies, $\alpha$-synucleinopathes, prion diseases, TDP-43 proteinopathies, respectively. Neuropathological examinations have demonstrated that these protein signatures often coexist, which is seen at a rate of $27-68 \%$ among the groups examined, according to several recent studies [1-3]. Among NDDs, Alzheimer's disease (AD) is the most common, and is the leading cause of dementia worldwide, representing 50-75\% of the cases [4]. It is also the disease in which the search for reliable fluid biomarkers has been the most successful, with the combination of decreased $A \beta 42$ (particularly when examined in a ratio with $A \beta 40$ ), and increased tau phosphorylated at threonine residue 181 (p-tau181) and T-tau in cerebrospinal fluid (CSF) constituting the typical CSF biomarker signature in $\mathrm{AD}$, which is now also included in the research criteria for $\mathrm{AD}$ [5]. However, because of the large majority of failed disappointing results from all clinical trials attempting to target $\mathrm{AD}$ progression, most clinical 
trials now attempt to target AD and other NDDs at a preclinical stage as well as more accurately characterizing the individuals included. One example highlighting the need of a reliable and scalable biological characterization of pathology is the recent publication of a paper by a consensus work group describing a recently discovered disease entity mimicking AD, limbicpredominant age-related transactive domain binding protein 43 (TDP-43) encephalopathy (LATE), which is present in $>20 \%$ of autopsies conducted in individuals $>80$ years old [6]. Therefore, the focus of much of current neurological biomarker research is to find reliable diagnostic and predictive biomarkers in more easily accessible biofluids, such as blood, at an early disease stage. Fortunately, research findings in recent years have generated promising results, which might soon be adopted in clinical and research settings, and will thus be the focus of this review together with recent progress in the field of CSF biomarkers. A summary of the biomarkers discussed can be found in figure 1. Since neurofilaments will be covered in other parts of this special issue, it will not be in the scope of this review.

\section{FLUID BIOMARKERS FOR AMYLOID PATHOLOGY}

Extracellular deposition of $A \beta$ into plaques, through the cleavage of APP with $\beta$-secretase 1 (BACE1) and $\gamma$-secretase is proposed as the main pathogenic event in AD [7]. Since the amyloid cascade hypothesis was presented three decades ago, it has resulted in increased understanding of AD pathogenesis, as well as in reliable diagnostic biomarkers in CSF and through amyloid positron emission tomography (PET) imaging, reflecting cerebral amyloid burden [8].

\section{$C S F$}

In patients with autosomal dominant $\mathrm{AD}$ from the Dominantly Inherited Alzheimer's Network (DIAN), longitudinal data suggests that low CSF A $\beta 42$ compared to non-carriers precedes expected symptomatic AD with 25 years, followed by abnormalities in brain glucose metabolism a decade later, further supporting the role of $\mathrm{A} \beta$ deposition as the earliest sign and initiator of disease pathology [9]. As previously mentioned, even though A $\beta 42$ in CSF is an widely accepted diagnostic test for $A \beta$ pathology in research criteria [5], as well as in the proposed biological staging of $\mathrm{AD}$ [10], with reduced CSF levels reflecting retention of $\mathrm{A} \beta 42$ in the brain parenchyma, research is still ongoing in terms of standardizing protocols for CSF analyses in clinical laboratory practice [11]. The field is now moving towards using A $\beta 42 / 40$ or 38 ratios, due to their ability to correct for inter-individual differences in amyloid processing and possible preanalytical confounders [12]. CSF A $\beta 42$ and A $\beta 42 / 40$ are highly concordant with A $\beta$ PET uptake [13]. However, in many cases, CSF A $\beta 42$ seemingly precedes A $\beta$ PET 
positivity [14] which reflects the ability of CSF to capture changes in oligomeric A $\beta$. Furthermore, in concordance with neuropathological findings that proteinopathies often coexist, A $\beta 42$ is decreased in most DLB patients [15] and many Parkinson disease (PD) dementia patients, but is normal in, e.g., frontotemporal dementia (FTD) patients [16].

\section{Blood}

In contrast to earlier reports [17], recent findings suggest that plasma A $\beta 42$, especially in ratio with $A \beta 40$, reflect cerebral $A \beta$ pathology. Current research demonstrates a good but not optimal correlation of plasma $\mathrm{A} \beta$ with cerebral $\mathrm{A} \beta$ pathology, as measured by PET or CSF. Promising results have been presented by several groups with immunoprecipitation mass spectrometry (IPMS) analyses [18] [19], as well as with immunoassays, such as Single molecule array (Simoa) [20, 21], enzyme-linked immunosorbent assay (ELISA) [22], and immunomagnetic reduction (IMR) assay [23, 24] (see Table 1). A recent validation study utilizing a fully automated immunoassay (Elecsys) to measure plasma $A \beta 42$ and $A \beta 40$ indicates the promising capabilities of plasma $A \beta$ in clinical laboratory practice [25]. However, the concordance between studies is poor, but slightly better when comparing mass spectrometric assays $[18,19]$. Immunoassays seemingly struggle with matrix effects possibly confounding the results, which are more effectively eliminated with IPMS techniques. This is reflected in the area under the receiver operation characteristics curve (AUC) ranging from 0.789-0.914. Schindler et al. report a difference between $A \beta$-positive and $A \beta$-negative of only 11 percent [19], this is compared to changes of around 50\% in CSF [26]. Small effect sizes between A $\beta$-positive and $A \beta$-negative individuals, possibly due to peripheral $A \beta$ expression [27], is an issue that needs to be addressed, if $A \beta$ is to be employed as a screening test for cerebral amyloidosis. To elucidate these issues, cooperative efforts such as round robins and validation studies are warranted. Furthermore, studies have also explored the possibility of measuring plasma $A \beta$ in heterogeneous forms [28] and exosomes [29], but data is still limited.

\section{TAU PATHOLOGY}

The aggregation of hyperphosphorylated forms of the axonal protein tau in the neuronal soma, forming neurofibrillary tangles (NFT), is a key neuropathological feature in AD, but tau inclusions in neurons or glial cells are also found in other NDDs [30]. 
The cornerstone markers T-tau and p-tau181 together with CSF A $\beta 42$ are proposed as biomarkers that biologically define AD [10]. They are considered diagnostic in the research criteria for AD [5]. T-tau is not different from HCs in most NDDs, except for AD and CJD [31]. However, CSF p-tau181 is seemingly AD-specific [32]. T-tau and p-tau181 correlates with tauPET in later stages of the disease, but seemingly not in cognitively unimpaired AD patients, indicating that CSF biomarkers may be used as diagnostic markers, but not reflecting disease stage [33]. However, other tau fragments have been investigated as biomarkers reflecting tangle pathology. Increased baseline levels of the N-terminal fragment cleaved at residue $224(\mathrm{~N}-224)$ distinguished $\mathrm{AD}$ from $\mathrm{HC}$ and patients with primary tauopathies, and predicted cognitive decline as well as conversion from MCI to AD [34]. A recent pilot study investigated a novel assay measuring C-terminally truncated (tau-368) tau found that lower levels of tau-368 was inversely correlated with disease stage, possibly indicating a decrease in tau production or more intriguingly sequestering of tau in NFTs [35]. It is important to note that this is a pilot study, meaning that these theories have to be further investigated. Additionally, recent data suggest that a novel assay targeting tau phosphorylated at 217 (p-tau217) very closely resembles the time of change and trajectory of p-tau181 in A $\beta$-positive individuals [36]. It is still not elucidated whether the increased p-tau181 and T-tau concentrations in CSF reflect tangle formation or neurodegeneration per se, as proposed in the research framework [10]. One study suggests that the increased CSF levels of tau are instead due to increased phosphorylation and secretion of tau in the CNS in response to brain amyloidosis [37]. Further, data from DIAN suggests that the increases of p-tau181 become less prominent in the symptomatic stages of the disease, and that this potentially reflects sequestering of phosphorylated tau in tangles [9]. This will be elucidated in further studies.

\section{Blood}

As previously stated, current research puts great effort in developing reliable screening and prognostic assays in blood for tau in NDDs, which has proven to be a difficult hurdle to overcome. These difficulties can partly be explained by the fact that tau is rapidly cleared from plasma (the apparent half-life of tau in blood is <24 hours), supported by studies conducted on patients with acute brain injury following cardiac arrest [38]. This is consistent with the findings that plasma T-tau correlates poorly with CSF T-tau [39]. Nevertheless, cross-sectional studies during the last 5 years have, in a fairly consistent manner, demonstrated that plasma T-tau levels 
are increased in patients with $\mathrm{AD}$ according to a meta-analysis [17], but do not differentiate significantly between MCI and HC [40, 41]. Studies have shown that plasma T-tau is associated with cognitive decline as well as risk of MCI [41, 42] and AD [43], and that it correlates negatively with grey matter density [44], but this remains to be examined in longitudinal studies. However, the overlap seems to be large across diagnostic groups, indicating that plasma T-tau alone is not suitable as a diagnostic marker for AD. As previously mentioned, tau pathology is also present in other NDDs and higher plasma levels of T-tau have been observed in FTD [45, 46], PD, vascular dementia (VaD) and Creutzfeldt-Jakob disease (CJD) [46], where levels are substantially increased compared with $\mathrm{HCs}$ and correlate strongly with rate of disease progression $[47,48]$. Targeting $\mathrm{N}$-terminal fragments also in plasma might be a way forward, with one study separating AD and MCI from HCs with great sensitivity and specificity albeit small sample sizes [49]. The recent reports of detection of p-tau181 in plasma has generated much interest, with three studies being able to distinguish AD from HCs, using Single molecule array (Simoa) [50], immunomagnetic reduction (IMR) [51] and Meso Scale Discovery (MSD) [52] technologies, respectively. While IMR data suggests increases in most NDDs, MSD studies clearly show specific increases only in the AD continuum corroborating CSF findings. Mielke et al. originally demonstrated a correlation between p-tau181, and amyloid/tau PET, which indicates that plasma p-tau181 is a good predictor of brain AD pathology. These findings were replicated in a recent study by Palmqvist et al., demonstrating that plasma p-tau181 associates with both A $\beta$ PET positivity as well as CSF p-tau181. Interestingly, the change in plasma p-tau181 became significant before amyloid PET, but after CSF/plasma A $\beta 42$ [36]. Thus, plasma p-tau181 might be useful both diagnostically as well as for disease staging. Nonetheless, validation studies in larger cohorts, as well as with other emerging assays, are warranted to determine the clinical utility of plasma p-tau181 as an AD marker for primary care settings, specialized centers and clinic trials.

\section{SYNAPTIC DEGENERATION}

Synaptic degeneration is an early event in AD and other NDDs [53], and synaptic loss is the best correlate of cognitive decline [54]. Abnormal magnetic resonance imaging (MRI) or fluorodeoxyglucose PET scans are currently proposed as markers of synaptic loss in the proposed staging of $\mathrm{AD} \mathrm{[10].} \mathrm{Another} \mathrm{emerging} \mathrm{method} \mathrm{to} \mathrm{detect} \mathrm{synaptic} \mathrm{loss} \mathrm{in} \mathrm{NDDs} \mathrm{is} \mathrm{PET}$ tracers targeting synaptic vesicle protein 2A (SV2A) [55]. In recent years, promising assays in biofluids targeting loss of synapses have been developed. 
Neurogranin is a postsynaptic protein involved in long-term potentiation and synaptic plasticity and increased concentrations in CSF in AD patients accurately predict cognitive decline from MCI to AD dementia [56]. These increases are neuropathologically associated with plaque pathology when comparing amyloid with tangle, synuclein and TDP-43 load [57]. These findings have been replicated by other groups [58, 59], supporting the use of CSF neurogranin as a biomarker to detect synaptic dysfunction in response to $\mathrm{A} \beta$ pathology. Recent data from the BioFINDER study indicates that CSF neurogranin increases significantly shortly after A $\beta$ accumulation, which further supports the idea that synaptic loss occurs early in AD patients [36]. Although initially thought to be AD-specific, a recent study demonstrated increased CSF neurogranin in CJD patients compared with cognitively unimpaired individuals and AD patients [60]. Other emerging biomarkers reflecting synaptic degeneration include synaptosomal-associated protein 25 (SNAP-25) [58, 61] and synaptotagmin-1 (SYT-1) [62], which are increased in AD and MCI compared with HC. Growth-associated protein 43 (GAP43 ) is also increased in AD compared to controls, and reflects amyloid and tau pathology, and seems to be AD-specific [63], although transient increases can be found after stroke [64]. Another emerging biomarker is neuronal pentraxin 2 (NPTX-2), which in one pilot study showed lower levels in AD, as well as in individuals with cognitive impairment [65].

\section{Blood}

No studies measuring neurogranin in plasma have yet been able to differentiate AD from HC, due to relatively high peripheral expression confounding a potential difference [66]. Exosomal enrichment might overcome this issue. Studies examining neuronally derived exosomal neurogranin in plasma have been able to separate $\mathrm{HCs}$ from $\mathrm{AD}[67,68]$, and stable MCI from MCI converting to AD [68], but more replication studies in this field are needed.

\section{ALPHA-SYNUCLEIN PATHOLOGY}

Misfolding of the $\alpha$-synuclein protein plays a major role in the development of common NDDs, such as PD and DLB, and other neuropathologically related disorders. It is also the main constituent of Lewy bodies, the signature protein inclusion found in PD and DLB. Therefore, much of the research on diagnostic markers for these diseases has been focused on identifying pathology-specific forms of the protein. 
It is relatively easy to measure total $\alpha$-synuclein concentration in CSF but its level does not correlate with Lewy body pathology. Instead, it is argued that high levels are a marker of neurodegeneration [69]. Additionally, a recent study in ADAD mutation carriers as well as sporadic AD patients demonstrated increased levels of total $\alpha$-synuclein and suggests that $\alpha$ synuclein is linked to $\mathrm{AD}$ pathophysiology through the $A P O E \varepsilon 4$ allele. Furthermore, total $\alpha-$ synuclein concentration in CSF increases if there is blood contamination of the sample, due to high expression in red blood cells, which further reduces its utility [70]. However, studies measuring oligomers and post translational modifications of $\alpha$-synuclein have shown at least slightly positive results, but still not satisfactory enough for clinical use (for review, see [69]). Nonetheless, total $\alpha$-synuclein in CSF might be used as a prognostic marker of motor progression in PD [71-73], and increased levels as a marker of synaptic degeneration [74]. Further, the idea of $\alpha$-synuclein oligomers spreading in a prion-like manner has sparked the idea that seeding aggregation assays, such as real-time quaking induced conversion (RT-QuIC) or protein-misfolding cyclic amplification (PMCA) could be methods to qualitatively detect pathological forms of $\alpha$-synuclein in CSF [75]. Studies analyzing CSF with RT-QuIC have been able to distinguish synucleinopathies from non-synucleinopathies with excellent diagnostic accuracy, detecting DLB and multiple system atrophy with 100\% and 80\% sensitivity, respectively [76]. Another study did a similar comparison and was able to discriminate between synucleinopathies (PD and DLB), and non-synucleinopathies with $100 \%$ specificity and $93 \%$ sensitivity [77]. Unsurprisingly, these assays did not discriminate between different synucleinopathies [77]. However, panels combining different biomarkers may be a way to differentiate PD from atypical parkinsonian disorders, with one combination reaching an AUC of 0.95 [74]. In conclusion, results from $\alpha$-synuclein aggregation assays are encouraging, but need to be validated in larger cohorts. There is also a need find reliable biomarker signatures to distinguish between different synucleinopathies.

\section{Blood}

Because of the high risk of contamination of plasma and serum samples with $\alpha$-synuclein from $\mathrm{RBC}[70]$, it is not a surprise that total $\alpha$-synuclein has yielded disappointing results as a diagnostic marker in whole blood, serum and plasma. However, better discriminatory power has been seen when instead measuring oligomeric or post-translationally modified $\alpha$-synuclein (for review, see [69]). The encouraging results from aggregation assays in CSF may prove to be effective in other biofluids. 


\section{GLIAL ACTIVATION AND NEUROINFLAMMATION}

Neuroinflammation, as well as activation of microglial cells and astrocytes, are key features of most NDDs, with most research having been performed in AD. During the last decade, it has been debated whether neuroinflammation and astrogliosis is an important driver of neurodegeneration, or a side effect of the accumulation of amyloid and tau.

\section{$C S F$}

A number of candidate markers have been examined in relation to inflammation/astroglial activation in NDDs, of which YKL-40, a glycoprotein expressed in both astrocytes and microglia, has proven to be maybe the most promising. Several cross-sectional, as well as longitudinal, studies in the past years have shown that CSF YKL-40 levels are modestly increased in patients with AD and FTD, but are relatively low in DLB, providing evidence for YKL-40 as a marker of neuroinflammation in the mentioned diseases [78, 79]. A study in the ADNI cohort showed conflicting results, with no significant separation of AD from HCs, but small sample sizes might not have given the power to detect a difference [58]. However, two recent longitudinal studies show that higher levels of YKL-40 in CSF correlate with cognitive decline as well as CSF tau levels [80, 81], and also with cortical thickness in CSF A $\beta$-positive individuals [81]. Increased levels in ADAD mutation carriers compared to non-carriers seem to appear 15 years before symptomatic disease is expected, together with markers of synaptic degeneration and neuronal injury [82]. A recent longitudinal study by Villar-Piqué et al. show higher plasma YKL-40 concentration among CJD patients compared with other NDs and HCs. This suggests that YKL-40 may be used as a marker of disease progression in CJD [83]. Lower levels of CSF YKL-40 compared to HCs have been observed in PD [84]. Other markers expressed in microglial cells that show promise is soluble triggering receptor expressed on microglial cells 2 (sTREM2), the secreted proteolytic degradation product of TREM2, which has been found at higher concentration in CSF from AD patients compared with HCs [85] with increases being observed already in individuals with reported subjective cognitive decline (SCD) [80]. Recently, the concentrations of CSF sTREM2 have been shown to be increased also in early symptomatic stages of sporadic $\mathrm{AD}[86,87]$. Interestingly, $\mathrm{A} \beta$ pathology and taurelated neurodegeneration may impact levels of CSF sTREM2 differently [87]. Data from the DIAN study of ADAD mutation carriers demonstrates that sTREM2 concentration in CSF increases in mutation carriers compared to non-carriers before symptomatic disease is expected, and after $\mathrm{A} \beta$ and T-tau [88]. Moreover, it has been shown that the concentrations of CSF sTREM2 vary between different disease-associated TREM2 genetic variant carriers [86, 87]. 
However, the highest CSF levels are found in patients with the autoimmune disease multiple sclerosis (MS) [89]. Other promising markers are monocyte chemoattractant protein (MCP-1) in CSF [80] and cytokine markers [81]. In conclusion, neuroinflammatory markers might be useful to pinpoint neuroinflammation in NDDs, which potentially could be of use as patient selection tools for clinical trials, in addition to core protein markers, or as a reflection of disease progression.

\section{Blood}

Some data suggest that YKL-40 is increased in plasma among patients with early AD [78, 90], but more studies are needed to confirm this finding. One study reported that higher plasma MCP-1 levels are associated with disease severity and faster cognitive decline in AD patients [91]. While sTREM2 is highly abundant in plasma and serum sTREM2 levels do not differ between multiple sclerosis, other inflammatory neurologic diseases and non-inflammatory controls [92]. Kleinberger et al. found no difference in plasma sTREM2 between healthy controls, AD and FTD [93]. Furthermore, a recent study indicates that there are no changes in plasma sTREM2 in TREM2 rare variants carriers (Ashton NJ et al., 2019 In Press). Studies suggesting such a change in TREM2 mRNA/protein levels among non-mutated TREM2 AD patients should therefore be carefully interpreted [94].

\section{TDP-43 PATHOLOGY}

Cytoplasmic inclusions of TDP-43 is implicated in a majority of patients with the motor neuron disease amyotrophic lateral sclerosis (ALS), FTD [95] as well LATE, a neuropathological disease entity clinically indistinguishable from AD [6].

\section{CSF and Blood}

Over the last ten years, proteomic studies targeting TDP-43 pathology have only generated modest results. CSF TDP-43 can be measured but is subjected to contamination with peripheral expression, and correlation with neuropathological findings are weak [96]. Both phosphorylated [97, 98] and total [99] plasma TDP-43 can be measured and show weak or no correlation with neuropathological examination. Due to the difficulties separating peripheral from CNS-derived or pathological forms of TDP-43, more work needs to be done to develop a biomarker for TDP-43 pathology.

\section{PRION PATHOLOGY}


The conversion of the physiological, cellular form of prion protein $\left(\mathrm{PrP}^{\mathrm{C}}\right)$ to the pathogenic, misfolded form of the protein (prion protein scrapie, $\mathrm{PrP}^{\mathrm{Sc}}$ ), together with extensive neuronal loss and spongiform appearance of the brain tissue at autopsy, characterizes this rare (incidence of 1 per million/year) group of fast progressing dementia, with CJD being the most common form.

\section{$C S F$}

Despite the difficulties of developing trustworthy specific immunoassays for the diagnosis of CJD and other prionopathies, the aggregation assay RT-QuIC has proven to be an effective method to detect $\mathrm{PrP}^{\mathrm{Sc}}$, which was first discovered in 2010 [100]. These results have since been replicated [101, 102], and according to a validation study, sporadic CJD was detected with a sensitivity of $92 \%$ and specificity of $100 \%$ using the method [103]. Among the patients who had a negative RT-QuIC result, 90\% where positive using another diagnostic method [103].

\section{Blood}

The sensitivity of this assay triggers the thought of RT-QuIC as a potential blood test for prion disease. However, studies have shown that blood contamination decreases the performance of the CSF assay by effectively inhibiting the aggregation cascade [104], which has decreased the interest in developing a blood-based assay for the diagnosis of CJD.

\section{NEURONAL INJURY}

Neurofilament light (NFL) is the major biomarker for neuronal degeneration and is increased in neurodegenerative, inflammatory, traumatic and vascular conditions [105]. Another promising biomarker reflecting neuronal injury in NDDs is the neuronal calcium sensor protein visinin-like protein 1 (VLP-1/VILIP-1). Increased concentrations of CSF VLP-1 in AD patients compared with HCs are a well replicated finding [17]. Recent longitudinal studies report increased VLP-1 levels in amyloid-positive MCI and AD patients compared with amyloidnegative MCI and HCs [58, 106], and a longitudinal decrease in AD patients [58], possibly reflecting extensive neuronal damage. VLP-1 also predicts progression from MCI to AD [106]. An association with future cognitive decline, hippocampal atrophy among amyloid-positive MCI and AD was also found [106]. In contrast to NFL, VLP-1 also differentiates between AD and non-AD dementias $[107,108]$. In conclusion, VLP-1 may be used in addition to NFL as a marker of neurodegeneration and disease progression in AD. Data on blood VLP-1 remains limited [107]. 


\section{FUTURE DIRECTIONS}

The rapid advancement in highly sensitive quantitative technologies has led to the promising translation of CSF biomarkers to blood and this includes A $\beta$, T-tau, p-tau and NFL. While studies on plasma A $\beta$ IPMS platforms show great promise in terms of diagnostic accuracy, it is a method with complexity, especially considering the desired use of plasma $A \beta$ as a screening tool with potential in primary care settings. Furthermore, no consensus cut-off values have been established yet, which is an important question to address. Additionally, there is still large interassay variability, and collaborative efforts are required to elucidate this. At AAIC 2019, there were different groups presenting plasma p-tau181 assays, reflecting tangle pathology, which is much needed for enrollment in clinical trials and future primary care screening procedures. Standardized measurements for these two core proteins would facilitate cheaper and more reliable subject inclusion in much warranted clinical trials. However, as previously mentioned, further investigations into what tau measurements in biofluids actually tell us are warranted. Moreover, since the overwhelming proportion of academic studies is performed in white Caucasian populations, it is not known how the assays developed perform in individuals of other ethnic backgrounds. Since an increasing proportion of people with NDDs are nonCaucasians, it is important to investigate how the available assays perform in these populations. Another important matter that needs a solution is the absence of reliable diagnostic biomarkers for major NDDs, such as TDP-43 pathology in FTD and ALS, and $\alpha$-synuclein pathology in PD and DLB. In conclusion, cooperative efforts are needed to validate the promising AD plasma assays available, as well as intensifying the search for biomarkers as reliable diagnostic tools for other NDDs.

\section{ACKNOWLEDGEMENTS}

NJA is funded by the Wallenburg Centre for Molecular and Translational. KB is supported by the Torsten Söderberg Foundation, Stockholm, Sweden. HZ is a Wallenberg Academy Fellow supported by grants from the Swedish Research Council (\#2018-02532), the European Research Council (\#681712), Swedish State Support for Clinical Research (\#ALFGBG720931) and the UK Dementia Research Institute at UCL. JS is supported by Mary Von Sydows donationsfond, and Gothenburg Medical Society (\#003-19). Figures were drawn by Nora Odquist.

\section{DECLARATIONS}


KB has served as a consultant or at advisory boards for Alector, Alzheon, CogRx, Biogen, Lilly, Novartis and Roche Diagnostics, and is a co-founder of Brain Biomarker Solutions in Gothenburg AB, a GU Venture-based platform company at the University of Gothenburg, all unrelated to the work presented in this paper. HZ has served at scientific advisory boards for Roche Diagnostics, Wave, Samumed and CogRx, has given lectures in symposia sponsored by Alzecure and Biogen, and is a co-founder of Brain Biomarker Solutions in Gothenburg AB, a GU Ventures-based platform company at the University of Gothenburg. JS and NJA have nothing to declare.

\section{HIGHLIGHTS}

- Protein biomarkers in cerebrospinal fluid now define Alzheimer's disease (AD).

- New ultrasensitive techniques have enabled promising replication in blood.

- This would enable the use of biomarkers to screen for AD in a primary care setting.

- The coexistence of pathologies complicates clinical trials and diagnostics of neurodegenerative diseases (NDDs).

- Biomarkers for NDDs that reflect other pathologies are greatly needed.

\section{REFERENCES}

1. Robinson, J.L., et al., Neurodegenerative disease concomitant proteinopathies are prevalent, age-related and APOE4-associated. Brain, 2018. 141(7): p. 2181-2193.

2. James, B.D., et al., TDP-43 stage, mixed pathologies, and clinical Alzheimer's-type dementia. Brain, 2016. 139(11): p. 2983-2993.

3. Beach, T.G., et al., Accuracy of the clinical diagnosis of Alzheimer disease at National Institute on Aging Alzheimer Disease Centers, 2005-2010. J Neuropathol Exp Neurol, 2012. 71(4): p. 266-73.

4. Winblad, B., et al., Defeating Alzheimer's disease and other dementias: a priority for European science and society. Lancet Neurol, 2016. 15(5): p. 455-532.

5. Dubois, B., et al., Advancing research diagnostic criteria for Alzheimer's disease: the IWG-2 criteria. Lancet Neurol, 2014. 13(6): p. 614-629.

6. $\quad$ Nelson, P.T., et al., Limbic-predominant age-related TDP-43 encephalopathy (LATE): consensus working group report. Brain, 2019. 142(6): p. 1503-1527.

7. Selkoe, D.J. and J. Hardy, The amyloid hypothesis of Alzheimer's disease at 25 years. EMBO Mol Med, 2016. 8(6): p. 595-608.

8. Ashton, N.J., et al., Update on biomarkers for amyloid pathology in Alzheimer's disease. Biomark Med, 2018. 12(7): p. 799-812.

*9. McDade, E., et al., Longitudinal cognitive and biomarker changes in dominantly inherited Alzheimer disease. Neurology, 2018. 91(14): p. e1295-e1306.

An article outlining the sequence and dynamics of some of the most important biomarkers for $\mathrm{AD}$ in a well-defined cohort of individuals with dominantly inherited AD. 
**10. Jack, C.R., Jr., et al., NIA-AA Research Framework: Toward a biological definition of Alzheimer's disease. Alzheimers Dement, 2018. 14(4): p. 535-562.

This article provides a model to define Alzheimer's Disease in living persons using biomarkers. The abbreviation used is AT(N), representing $\beta$ amyloid, pathologic tau and neurodegeneration respectively. It should be interpreted as an attempt to coordinate AD biomarker research.

11. Janelidze, S., et al., Towards a unified protocol for handling of CSF before betaamyloid measurements. Alzheimers Res Ther, 2019. 11(1): p. 63.

12. Hansson, O., et al., Advantages and disadvantages of the use of the CSF Amyloid beta (Abeta) 42/40 ratio in the diagnosis of Alzheimer's Disease. Alzheimers Res Ther, 2019. 11(1): p. 34.

13. Hansson, O., et al., CSF biomarkers of Alzheimer's disease concord with amyloid-beta PET and predict clinical progression: A study of fully automated immunoassays in BioFINDER and ADNI cohorts. Alzheimers Dement, 2018. 14(11): p. 1470-1481.

14. Mattsson, N., et al., Staging beta-Amyloid Pathology With Amyloid Positron Emission Tomography. JAMA Neurol, 2019.

15. van Steenoven, I., et al., Cerebrospinal Fluid Alzheimer's Disease Biomarkers Across the Spectrum of Lewy Body Diseases: Results from a Large Multicenter Cohort. J Alzheimers Dis, 2016. 54(1): p. 287-95.

16. Ewers, M., et al., CSF biomarkers for the differential diagnosis of Alzheimer's disease: A large-scale international multicenter study. Alzheimers Dement, 2015. 11(11): p. 1306-15.

17. Olsson, B., et al., CSF and blood biomarkers for the diagnosis of Alzheimer's disease: a systematic review and meta-analysis. Lancet Neurol, 2016. 15(7): p. 673-684.

**18. Nakamura, A., et al., High performance plasma amyloid-beta biomarkers for Alzheimer's disease. Nature, 2018. 554(7691): p. 249-254.

This article was the first method accurately predicting brain amyloid status by PET and CSF with plasma amyloid measurements using IPMS method in healthy controls, MCI and AD.

**19. Schindler, S.E., et al., High-precision plasma beta-amyloid 42/40 predicts current and future brain amyloidosis. Neurology, 2019.

This article provides evidence that plasma amyloid measurements using LCMS method predicts amyloid status by PET and CSF. This method has potential use in disease prevention trials.

20. Janelidze, S., et al., Plasma beta-amyloid in Alzheimer's disease and vascular disease. Sci Rep, 2016. 6: p. 26801.

*21. Verberk, I.M.W., et al., Plasma Amyloid as Prescreener for the Earliest Alzheimer Pathological Changes. Ann Neurol, 2018. 84(5): p. 648-658.

This article presents evidence that plasma amyloid measurements using single molecule array (Simoa) immunoassay technology predicts amyloid status by PET and CSF in individuals with subjective cognitive decline and risk of progressing to MCI and AD. 
22. Risacher, S.L., et al., Plasma amyloid beta levels are associated with cerebral amyloid and tau deposition. Alzheimers Dement (Amst), 2019. 11: p. 510-519.

23. Teunissen, C.E., et al., Plasma Amyloid-beta (Abeta42) Correlates with Cerebrospinal Fluid Abeta42 in Alzheimer's Disease. J Alzheimers Dis, 2018. 62(4): p. 1857-1863.

24. Fan, L.-Y., et al., The Relation Between Brain Amyloid Deposition, Cortical Atrophy, and Plasma Biomarkers in Amnesic Mild Cognitive Impairment and Alzheimer's Disease. Frontiers in Aging Neuroscience, 2018. 10(175).

**25. Palmqvist, S., et al., Performance of Fully Automated Plasma Assays as Screening Tests for Alzheimer Disease-Related beta-Amyloid Status. JAMA Neurol, 2019.

This is the first article successfully measuring plasma amyloid using a fully automated assay to predict amyloid status by PET and CSF, which has great potential to be used in a clinical setting.

26. Blennow, K. and E. Vanmechelen, CSF markers for pathogenic processes in Alzheimer's disease: diagnostic implications and use in clinical neurochemistry. Brain Res Bull, 2003. 61(3): p. 235-42.

27. Citron, M., et al., Excessive production of amyloid beta-protein by peripheral cells of symptomatic and presymptomatic patients carrying the Swedish familial Alzheimer disease mutation. Proc Natl Acad Sci U S A, 1994. 91(25): p. 11993-7.

*28. Kim, Y., et al., Comparative analyses of plasma amyloid-beta levels in heterogeneous and monomerized states by interdigitated microelectrode sensor system. Sci Adv, 2019. 5(4): p. eaav1388.

This article desribes an assay measuring amyloid-beta in different states, and provides evidence that it correlates with PET amyloid status.

*29. Lim, C.Z.J., et al., Subtyping of circulating exosome-bound amyloid beta reflects brain plaque deposition. Nat Commun, 2019. 10(1): p. 1144.

This article desribes an assay measuring amyloid-beta in circulating exosomes, displaying a correlation with brain amyloid status by PET.

30. Irwin, D.J., Tauopathies as clinicopathological entities. Parkinsonism Relat Disord, 2016. 22 Suppl 1: p. S29-33.

31. Skillback, T., et al., Diagnostic performance of cerebrospinal fluid total tau and phosphorylated tau in Creutzfeldt-Jakob disease: results from the Swedish Mortality Registry. JAMA Neurol, 2014. 71(4): p. 476-83.

32. Sjogren, M., et al., CSF levels of tau, beta-amyloid(1-42) and GAP-43 in frontotemporal dementia, other types of dementia and normal aging. $\mathrm{J}$ Neural Transm (Vienna), 2000. 107(5): p. 563-79.

33. Scholl, M., et al., Biomarkers for tau pathology. Mol Cell Neurosci, 2019. 97: p. 1833.

*34. Cicognola, C., et al., Novel tau fragments in cerebrospinal fluid: relation to tangle pathology and cognitive decline in Alzheimer's disease. Acta Neuropathol, 2019. 137(2): p. 279-296.

The article provides evidence of tau being present as fragments in CSF, which can be used to discriminate $\mathrm{AD}$ from controls and other tauopathies. 
35. Leuzy, A., et al., Longitudinal tau and metabolic PET imaging in relation to novel CSF tau measures in Alzheimer's disease. Eur J Nucl Med Mol Imaging, 2019. 46(5): p. 1152-1163.

**36. Palmqvist, S., et al., Cerebrospinal fluid and plasma biomarker trajectories with increasing amyloid deposition in Alzheimer's disease. EMBO Mol Med, 2019: p. e11170.

This article investigated the trajectories of established biomarkers for AD in both plasma and CSF based on amyloid positivity by PET on different platforms, which is the first study to do so. It also provides evidence that neurogranin among other markers changes very early in the process of amyloid accumulation.

**37. Sato, C., et al., Tau Kinetics in Neurons and the Human Central Nervous System. Neuron, 2018. 98(4): p. 861-864.

In this article, studying tau kinetics in human induced pluripotent stem cell (iPSC)-derived neuron, it is suggested that there is a link between amyloid plaque burdain and tau physiology.

38. Randall, J., et al., Tau proteins in serum predict neurological outcome after hypoxic brain injury from cardiac arrest: results of a pilot study. Resuscitation, 2013. 84(3): p. 351-6.

39. Pereira, J.B., et al., Association between cerebrospinal fluid and plasma neurodegeneration biomarkers with brain atrophy in Alzheimer's disease. Neurobiology of Aging, 2017. 58: p. 14-29.

40. Dage, J.L., et al., Levels of tau protein in plasma are associated with neurodegeneration and cognitive function in a population-based elderly cohort. Alzheimers Dement, 2016. 12(12): p. 1226-1234.

41. Mattsson, N., et al., Plasma tau in Alzheimer disease. Neurology, 2016. 87(17): p. 1827-1835.

42. Mielke, M.M., et al., Association of Plasma Total Tau Level With Cognitive Decline and Risk of Mild Cognitive Impairment or Dementia in the Mayo Clinic Study on Aging. JAMA Neurol, 2017. 74(9): p. 1073-1080.

*43. Pase, M.P., et al., Assessment of Plasma Total Tau Level as a Predictive Biomarker for Dementia and Related Endophenotypes. JAMA Neurol, 2019.

The findings in this article provide evidence that plasma total tau levels can predict future dementia and can be used as a risk stratification tool in prevention trials.

44. Deters, K.D., et al., Plasma Tau Association with Brain Atrophy in Mild Cognitive Impairment and Alzheimer's Disease. J Alzheimers Dis, 2017. 58(4): p. 1245-1254.

45. Foiani, M.S., et al., Plasma tau is increased in frontotemporal dementia. J Neurol Neurosurg Psychiatry, 2018. 89(8): p. 804-807.

46. Yang, S.Y., et al., Analytical performance of reagent for assaying tau protein in human plasma and feasibility study screening neurodegenerative diseases. Sci Rep, 2017. 7(1): p. 9304.

47. Staffaroni, A.M., et al., Association of Blood and Cerebrospinal Fluid Tau Level and Other Biomarkers With Survival Time in Sporadic Creutzfeldt-Jakob Disease. JAMA Neurol, 2019. 
48. Thompson, A.G.B., et al., Neurofilament light chain and tau concentrations are markedly increased in the serum of patients with sporadic Creutzfeldt-Jakob disease, and tau correlates with rate of disease progression. J Neurol Neurosurg Psychiatry, 2018. 89(9): p. 955-961.

49. Chen, Z., et al., Learnings about the complexity of extracellular tau aid development of a blood-based screen for Alzheimer's disease. Alzheimers Dement, 2019. 15(3): p. 487-496.

50. Tatebe, H., et al., Quantification of plasma phosphorylated tau to use as a biomarker for brain Alzheimer pathology: pilot case-control studies including patients with Alzheimer's disease and down syndrome. Mol Neurodegener, 2017. 12(1): p. 63.

51. Yang, C.C., et al., Assay of Plasma Phosphorylated Tau Protein (Threonine 181) and Total Tau Protein in Early-Stage Alzheimer's Disease. J Alzheimers Dis, 2018. 61(4): p. 1323-1332.

**52. Mielke, M.M., et al., Plasma phospho-tau181 increases with Alzheimer's disease clinical severity and is associated with tau- and amyloid-positron emission tomography. Alzheimers Dement, 2018. 14(8): p. 989-997.

This article investigated plasma p-tau181 in AD dementia compared to $\mathrm{CU}$, and found that higher levels were present in those with $\mathrm{AD}$ compared to $\mathrm{CU}$, and those with positive tau and A $\beta$ PET.

53. Masliah, E., et al., Altered expression of synaptic proteins occurs early during progression of Alzheimer's disease. Neurology, 2001. 56(1): p. 127-9.

54. Blennow, K., et al., Synaptic pathology in Alzheimer's disease: relation to severity of dementia, but not to senile plaques, neurofibrillary tangles, or the ApoE4 allele. $\mathrm{J}$ Neural Transm (Vienna), 1996. 103(5): p. 603-18.

55. Chen, M.K., et al., Assessing Synaptic Density in Alzheimer Disease With Synaptic Vesicle Glycoprotein $2 A$ Positron Emission Tomographic Imaging. JAMA Neurol, 2018. 75(10): p. 1215-1224.

56. Kvartsberg, H., et al., Characterization of the postsynaptic protein neurogranin in paired cerebrospinal fluid and plasma samples from Alzheimer's disease patients and healthy controls. Alzheimers Res Ther, 2015. 7(1): p. 40.

*57. Portelius, E., et al., Cerebrospinal fluid neurogranin concentration in neurodegeneration: relation to clinical phenotypes and neuropathology. Acta Neuropathol, 2018. 136(3): p. 363-376.

This study provide evidence that the synaptic protein neurogranin is seemingly AD specific, when compared with other common neurodegenerative diseases.

58. Sutphen, C.L., et al., Longitudinal decreases in multiple cerebrospinal fluid biomarkers of neuronal injury in symptomatic late onset Alzheimer's disease. Alzheimers Dement, 2018. 14(7): p. 869-879.

59. Tarawneh, R., et al., Diagnostic and Prognostic Utility of the Synaptic Marker Neurogranin in Alzheimer Disease. JAMA Neurol, 2016. 73(5): p. 561-71.

60. Blennow, K., et al., CSF neurogranin as a neuronal damage marker in CJD: a comparative study with AD. J Neurol Neurosurg Psychiatry, 2019.

61. Zhang, H., et al., Cerebrospinal fluid synaptosomal-associated protein 25 is a key player in synaptic degeneration in mild cognitive impairment and Alzheimer's disease. Alzheimers Research \& Therapy, 2018. 10. 
62. Ohrfelt, A., et al., The pre-synaptic vesicle protein synaptotagmin is a novel biomarker for Alzheimer's disease. Alzheimers Res Ther, 2016. 8(1): p. 41.

*63. Sandelius, A., et al., Elevated CSF GAP-43 is Alzheimer's disease specific and associated with tau and amyloid pathology. Alzheimers Dement, 2019. 15(1): p. 5564.

This study provides evidence that the synaptic protein GAP-43 is increased in neuropathologically confirmed AD cases, and that it accurately distinguishes AD from other pathologies.

64. Sandelius, A., et al., Transient increase in CSF GAP-43 concentration after ischemic stroke. BMC Neurol, 2018. 18(1): p. 202.

65. Xiao, M.F., et al., NPTX2 and cognitive dysfunction in Alzheimer's Disease. Elife, 2017. 6.

66. De Vos, A., et al., C-terminal neurogranin is increased in cerebrospinal fluid but unchanged in plasma in Alzheimer's disease. Alzheimers Dement, 2015. 11(12): p. 1461-1469.

67. Goetzl, E.J., et al., Decreased synaptic proteins in neuronal exosomes of frontotemporal dementia and Alzheimer's disease. FASEB J, 2016. 30(12): p. 41414148.

68. Winston, C.N., et al., Prediction of conversion from mild cognitive impairment to dementia with neuronally derived blood exosome protein profile. Alzheimers Dement (Amst), 2016. 3: p. 63-72.

*69. Parnetti, L., et al., CSF and blood biomarkers for Parkinson's disease. Lancet Neurol, 2019. 18(6): p. 573-586.

This is a key review of biomarkers for Parkinson's Disease summarizing different markers and potential context of use.

70. Barbour, R., et al., Red blood cells are the major source of alpha-synuclein in blood. Neurodegener Dis, 2008. 5(2): p. 55-9.

71. Pagano, G., et al., REM behavior disorder predicts motor progression and cognitive decline in Parkinson disease. Neurology, 2018. 91(10): p. e894-e905.

72. Hall, S., et al., CSF biomarkers and clinical progression of Parkinson disease. Neurology, 2015. 84(1): p. 57-63.

73. Majbour, N.K., et al., Longitudinal changes in CSF alpha-synuclein species reflect Parkinson's disease progression. Mov Disord, 2016. 31(10): p. 1535-1542.

74. Magdalinou, N.K., et al., A panel of nine cerebrospinal fluid biomarkers may identify patients with atypical parkinsonian syndromes. J Neurol Neurosurg Psychiatry, 2015. 86(11): p. 1240-7.

*75. Paciotti, S., et al., Are We Ready for Detecting alpha-Synuclein Prone to Aggregation in Patients? The Case of "Protein-Misfolding Cyclic Amplification" and "Real-Time Quaking-Induced Conversion" as Diagnostic Tools. Frontiers in Neurology, 2018. 9.

This review discusses the potential of using aggregation assays to for the diagnosis of synucleinopathies, which has attracted much interest lately.

*76. Shahnawaz, M., et al., Development of a Biochemical Diagnosis of Parkinson Disease by Detection of alpha-Synuclein Misfolded Aggregates in Cerebrospinal Fluid. JAMA Neurol, 2017. 74(2): p. 163-172. 
This study analyzing CSF with RT-QuIC provide evidence that synucleinopathies can be distinguished from non-synucleinopathies with excellent diagnostic accuracy.

77. Groveman, B.R., et al., Rapid and ultra-sensitive quantitation of disease-associated alpha-synuclein seeds in brain and cerebrospinal fluid by alphaSyn RT-QuIC. Acta Neuropathol Commun, 2018. 6(1): p. 7.

78. Craig-Schapiro, R., et al., YKL-40: a novel prognostic fluid biomarker for preclinical Alzheimer's disease. Biol Psychiatry, 2010. 68(10): p. 903-12.

**79. Janelidze, S., et al., Cerebrospinal fluid neurogranin and YKL-40 as biomarkers of Alzheimer's disease. Ann Clin Transl Neurol, 2016. 3(1): p. 12-20.

In this study, it is concluded that several neuroinflammatory markers are increased with cortical thinning in AD patients, and are associated with higher risk to develop dementia among cognitively unimpaired individuals.

80. Nordengen, K., et al., Glial activation and inflammation along the Alzheimer's disease continuum. J Neuroinflammation, 2019. 16(1): p. 46.

81. Janelidze, S., et al., CSF biomarkers of neuroinflammation and cerebrovascular dysfunction in early Alzheimer disease. Neurology, 2018. 91(9): p. e867-e877.

*82. Schindler, S.E., et al., Emerging cerebrospinal fluid biomarkers in autosomal dominant Alzheimer's disease. Alzheimers Dement, 2019. 15(5): p. 655-665.

This study investigated less established biomarkers for AD in ADAD patients, and demonstrated that many of these markers changes several years before expected symptomatic disease.

*83. Villar-Pique, A., et al., Plasma YKL-40 in the spectrum of neurodegenerative dementia. J Neuroinflammation, 2019. 16(1): p. 145.

This study concludes that YKL-40 is also increased in patients with CJD, which has not been demonstrated before.

84. Hall, S., et al., Cerebrospinal fluid concentrations of inflammatory markers in Parkinson's disease and atypical parkinsonian disorders. Sci Rep, 2018. 8(1): p. 13276.

85. Suarez-Calvet, M., et al., sTREM2 cerebrospinal fluid levels are a potential biomarker for microglia activity in early-stage Alzheimer's disease and associate with neuronal injury markers. EMBO Mol Med, 2016. 8(5): p. 466-76.

86. Piccio, L., et al., Cerebrospinal fluid soluble TREM2 is higher in Alzheimer disease and associated with mutation status. Acta Neuropathol, 2016. 131(6): p. 925-33.

*87. Suarez-Calvet, M., et al., Early increase of CSF sTREM2 in Alzheimer's disease is associated with tau related-neurodegeneration but not with amyloid-beta pathology. Mol Neurodegener, 2019. 14(1): p. 1.

This study shows that tau-related neurodegeneration is associated with an increase in CSF sTREM2, while A $\beta$ pathology in the absence of downstream tau-related neurodegeneration is associated with a decrease in CSF sTREM2. 
88. Suarez-Calvet, M., et al., Early changes in CSF sTREM2 in dominantly inherited Alzheimer's disease occur after amyloid deposition and neuronal injury. Sci Transl Med, 2016. 8(369): p. 369ra178.

89. Ohrfelt, A., et al., Soluble TREM-2 in cerebrospinal fluid from patients with multiple sclerosis treated with natalizumab or mitoxantrone. Mult Scler, 2016. 22(12): p. 15871595.

90. Choi, J., H.W. Lee, and K. Suk, Plasma level of chitinase 3-like 1 protein increases in patients with early Alzheimer's disease. J Neurol, 2011. 258(12): p. 2181-5.

*91. Lee, W.J., et al., Plasma MCP-1 and Cognitive Decline in Patients with Alzheimer's Disease and Mild Cognitive Impairment: A Two-year Follow-up Study. Sci Rep, 2018. 8(1): p. 1280.

To our knowledge, this is the first study reporting that higher plasma MCP-1 levels are associated with disease severity and faster cognitive decline in AD patients.

92. Piccio, L., et al., Identification of soluble TREM-2 in the cerebrospinal fluid and its association with multiple sclerosis and CNS inflammation. Brain, 2008. 131(Pt 11): p. 3081-91.

93. Kleinberger, G., et al., TREM2 mutations implicated in neurodegeneration impair cell surface transport and phagocytosis. Sci Transl Med, 2014. 6(243): p. $243 \mathrm{ra} 86$.

94. $\mathrm{Hu}, \mathrm{N}$., et al., Increased expression of TREM2 in peripheral blood of Alzheimer's disease patients. J Alzheimers Dis, 2014. 38(3): p. 497-501.

95. Neumann, M., et al., Ubiquitinated TDP-43 in frontotemporal lobar degeneration and amyotrophic lateral sclerosis. Science, 2006. 314(5796): p. 130-3.

96. Steinacker, P., et al., TDP-43 in cerebrospinal fluid of patients with frontotemporal lobar degeneration and amyotrophic lateral sclerosis. Arch Neurol, 2008. 65(11): p. 1481-7.

97. Foulds, P.G., et al., Plasma phosphorylated-TDP-43 protein levels correlate with brain pathology in frontotemporal lobar degeneration. Acta Neuropathologica, 2009. 118(5): p. 647-658.

98. Suarez-Calvet, M., et al., Plasma phosphorylated TDP-43 levels are elevated in patients with frontotemporal dementia carrying a C9orf72 repeat expansion or a GRN mutation. J Neurol Neurosurg Psychiatry, 2014. 85(6): p. 684-91.

99. Foulds, $\mathrm{P}$., et al., TDP-43 protein in plasma may index TDP-43 brain pathology in Alzheimer's disease and frontotemporal lobar degeneration. Acta Neuropathologica, 2008. 116(2): p. 141-146.

100. Atarashi, R., et al., Ultrasensitive human prion detection in cerebrospinal fluid by real-time quaking-induced conversion. Nat Med, 2011. 17(2): p. 175-8.

101. Bongianni, M., et al., Diagnosis of Human Prion Disease Using Real-Time QuakingInduced Conversion Testing of Olfactory Mucosa and Cerebrospinal Fluid Samples. JAMA Neurol, 2017. 74(2): p. 155-162.

102. Satoh, K., R. Atarashi, and N. Nishida, Real-Time Quaking-Induced Conversion for Diagnosis of Prion Disease. Methods Mol Biol, 2017. 1658: p. 305-310.

*103. Green, A.J.E., RT-QuIC: a new test for sporadic CJD. Pract Neurol, 2019. 19(1): p. 49-55.

This clinical validation study reported that sporadic CJD was detected by RT-QuIC with a sensitivity of $92 \%$ and specificity of $100 \%$ using the method, which is an important finding for the clinical diagnosis of CJD. 
104. Foutz, A., et al., Diagnostic and prognostic value of human prion detection in cerebrospinal fluid. Ann Neurol, 2017. 81(1): p. 79-92.

105. Gaetani, L., et al., Neurofilament light chain as a biomarker in neurological disorders. J Neurol Neurosurg Psychiatry, 2019. 90(8): p. 870-881.

*106. Zhang, H., et al., Cerebrospinal fluid phosphorylated tau, visinin-like protein-1, and chitinase-3-like protein 1 in mild cognitive impairment and Alzheimer's disease. Transl Neurodegener, 2018. 7: p. 23.

This article provides evidence that VLP-1, among other proteins, is found in higher levels in patients with $\mathrm{AD}$, and reflects disease progression.

107. Tarawneh, R., et al., Visinin-like protein-1: diagnostic and prognostic biomarker in Alzheimer disease. Ann Neurol, 2011. 70(2): p. 274-85.

108. Babic Leko, M., et al., Predictive Value of Cerebrospinal Fluid Visinin-Like Protein-1 Levels for Alzheimer's Disease Early Detection and Differential Diagnosis in Patients with Mild Cognitive Impairment. J Alzheimers Dis, 2016. 50(3): p. 765-78. 


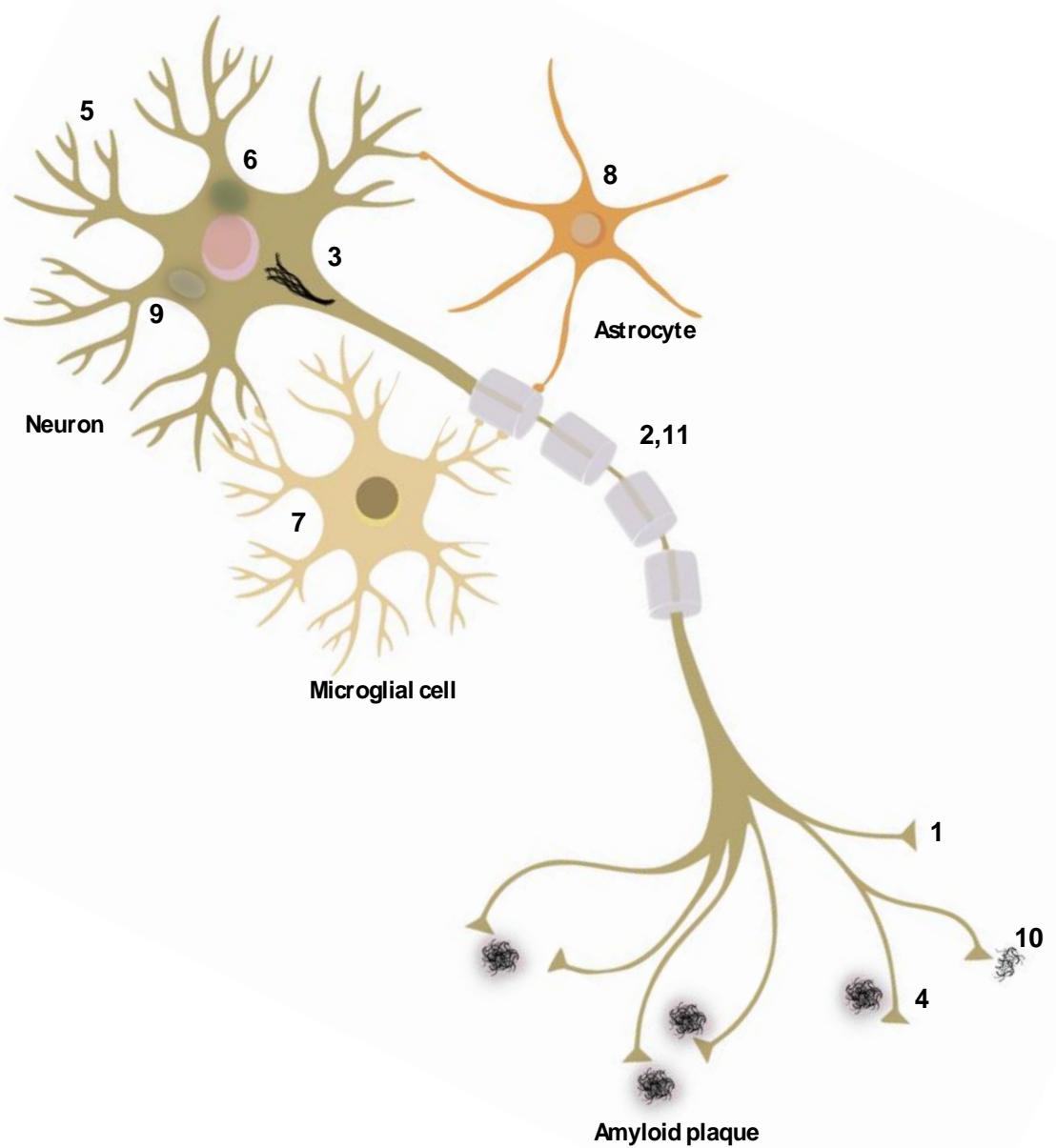

Amyloid pathology

1. $A \beta 42, A \beta 40, A \beta 38, A \beta$ oligomers

Tau pathology

2. T-tau

3. P181-tau in neurofibrillary tangles

Synaptic degeneration

4. SNAP-25, SYT-1,

5. GAP-43, neurogranin, NPTX-2

$\boldsymbol{\alpha}$-Synuclein pathology

6. $\alpha$-Synuclein inclusion

Glial activation and neuroinflammation

7. TREM-2, MCP-1, YKL-40, cytokines

8. YKL-40, cytokines

TDP-43 pathology

9. TDP-43 inclusion (no marker available)

Prion pathology

10. PrPSC aggregation

Neuronal injury

11. VLP-1

ers and localization of 


\begin{tabular}{|c|c|c|c|c|c|}
\hline Method & $\begin{array}{l}\text { Biomarkers measured in } \\
\text { plasma }\end{array}$ & $\begin{array}{l}\text { Change in Amyloid } \\
\text { (A) + VS A-group }\end{array}$ & $\begin{array}{c}\text { Receiver } \\
\text { Operating } \\
\text { Characteristic } \\
\text { (ROC) (A+ VS A- as } \\
\text { diagnosed by PET } \\
\text { and CSF) }\end{array}$ & $\begin{array}{l}\text { Correlation of plasma biomarkers with } \\
\text { PET (Mean Cortical Standard Uptake } \\
\text { Value Ratio (mSUVR)) and CSF }\end{array}$ & Reference \\
\hline $\begin{array}{l}\text { IP-MS } \\
\text { (LC/MS) }\end{array}$ & 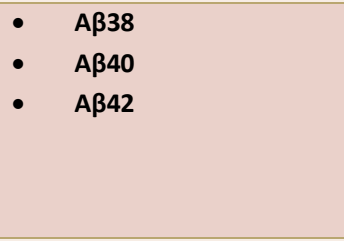 & $\begin{array}{l}\text { - } A \beta 42: \downarrow \text { in } A+ \\
\text { group } \\
\text { A } \$ \text { 42/ A } \beta 40 \text { ratio: } \\
\downarrow \text { in A+ group }\end{array}$ & $\begin{array}{l}\text { PET } \\
A \beta 42 / \quad A \beta 40 \text { ratio: } \\
A \cup C=0.88 \\
\text { CSF } \\
\text { (p-tau181/A } 342 \text { ) } \\
A \beta 42 / A \beta 40 \text { ratio: } \\
A \cup C=0.85\end{array}$ & $\begin{array}{l}\text { PET } \\
A \beta 40 / A \beta 42 \text { ratio: } r=-0,55 \\
C S F \\
\text { Plasma } A \beta 42 / A \beta 40 \text { ratio and } C S F A \beta 42 / A \beta 40: r= \\
0,66\end{array}$ & [19] \\
\hline $\begin{array}{c}\text { IP-MS } \\
\text { (MALDI-TOF) }\end{array}$ & $\begin{array}{ll}- & A \beta 40 \\
\text { - } & A \beta 42 \\
\text { - } & A P P 669-711\end{array}$ & $\begin{array}{ll}\text { - } & \text { A } 342: \downarrow \text { in A+ } \\
\text { group } \\
\text { - } \quad \text { A } 440 / A \beta 42 \text { ratio: } \\
\text { - in A+ group } \\
\text { - } \quad \text { APP669-711/ AB42 } \\
\text { ratio: } \uparrow \text { in A+ } \\
\text { group } \\
\text { Composite } \\
\text { biomarker: } \uparrow \text { in } \\
\text { A+ group } \\
\text { Composite biomarker } \\
\text { equals average of the } \\
\text { normalized APP669/711: } \\
\text { A642 ratio and A640/ } \\
\text { A642 ratio }\end{array}$ & $\begin{array}{l}P E T \\
A \beta 42: A U C=0.789 \\
A B 40 / A \beta 42 \text { ratio: } \\
A U C=0.886 \\
\text { APP669-711/A } 442 \\
\text { ratio: } A \cup C=0.861 \\
\text { Composite biomarker: } \\
\text { AUC=0.914 } \\
\text { CSF } \\
\text { AIBL sub-group }(n=46) \\
\text { Composite biomarker: } \\
\text { AUC }=0.876\end{array}$ & 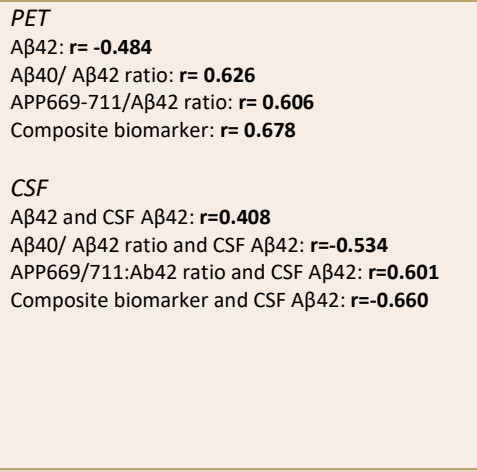 & [18] \\
\hline ELISA & $\begin{array}{ll}- & A \beta 40 \text { (total and free) } \\
\text { - } & A \beta 42 \text { (total and free) }\end{array}$ & $\begin{array}{l}\text { TA } \quad \text { 42/ TA } 40 \\
\text { ratio: } \downarrow \text { in A+ } \\
\text { group }\end{array}$ & $\begin{array}{l}\text { PET } \\
\text { TAB40/ TAB42 ratio } \\
\text { AUC }=\mathbf{0 . 7 7 5}\end{array}$ & $\begin{array}{l}\text { PET (mean global cortical amyloid SUVR) } \\
\text { TA } \beta 40 / \text { TA } \beta 42: r=-0,514\end{array}$ & [22] \\
\hline ELECSYS & $\begin{array}{l}\text { - } \\
\text { - }\end{array}$ & $\begin{array}{ll}- & \mathbf{A} \beta 42: \downarrow \text { in } A+ \\
& \text { group } \\
\text { - } & \mathbf{A} \beta 42 / A \beta 40 \text { ratio: } \\
& \downarrow \text { in A+ group }\end{array}$ & $\begin{array}{l}C S F \\
A \beta 42: A U C=0.71 \\
A \beta 42 / A \beta 40 \text { ratio: } \\
A \cup C=0.77 \\
A \beta 42, A \beta 40: A \cup C= \\
0.80\end{array}$ & $\begin{array}{l}\text { CSF } \\
\text { Plasma } A \beta 42 \text { and CSF } A \beta 42: r=0.373 \\
\text { Plasma } A \beta 42 / A \beta 40 \text { ratio and } C S F A \beta 42 / A \beta 40: \\
r=0.476 \\
\text { Plasma } A \beta 40 \text { ratio and CSF } A \beta 40: r=0.100\end{array}$ & [25] \\
\hline SIMOA & $\begin{array}{l}\text { - } \\
\text { - }\end{array}$ & $\begin{array}{l}\text { - } A \beta 42: \downarrow \text { in } A+ \\
\text { group } \\
\text { A } \$ 42 / A \beta 40 \text { ratio: } \\
\downarrow \text { in } A+\text { group } \\
\end{array}$ & $\begin{array}{l}P E T \\
A \beta 42: A U C=0.604 \\
A \beta 42 / A \beta 40 \text { ratio: } A \cup C \\
=0.621 \\
C S F \\
A \beta 42: A \cup C=0.655 \\
A \beta 42 / A \beta 40 \text { ratio: } A \cup C \\
=0.683\end{array}$ & $\begin{array}{l}P E T \\
A \beta 42: r=-0.162 \\
A \beta 40: r=-0.012 \\
A \beta 42 / A \beta 40 \text { ratio: } r=-0.167 \\
C S F \\
\text { Plasma } A \beta 42 \text { and CSF } A \beta 42: r=0.274 \\
\text { Plasma } A \beta 42 / A \beta 40 \text { ratio and } C S F A \beta 42 / A \beta 40 \text { : } \\
r=0.215 \\
\text { Plasma } A \beta 40 \text { ratio and } C S F A \beta 40: r=0.136\end{array}$ & [20] \\
\hline SIMOA & $\begin{array}{l}-A \beta 40 \\
-\quad A \beta 42\end{array}$ & 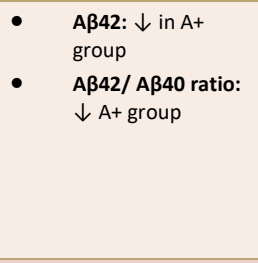 & $\begin{array}{l}P E T \\
A \beta 42: A U C=0.66 \\
A \beta 42 / A \beta 40 \text { ratio: } A \cup C \\
=0.68 \\
C S F \\
A B 42: A U C=0.66 \\
A B 42 / A \beta 40 \text { ratio: } A \cup C \\
=0.77\end{array}$ & $\begin{array}{l}\text { CSF } \\
\text { Plasma } A \beta 42 \text { and CSF } A \beta 42: r=0.18 \\
\text { Plasma } A \beta 42 / A \beta 40 \text { ratio and } C S F A \beta 42: r=0.38\end{array}$ & [21] \\
\hline IMR & - $\quad A \beta 42$ & 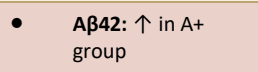 & & $\begin{array}{l}\text { CSF } \\
\text { Plasma } A \beta 42 \text { and CSF } A \beta 42 r=-0.352\end{array}$ & [23] \\
\hline \multirow[t]{3}{*}{ IMR } & $\begin{array}{l}\text { - } \\
\text { - }\end{array}$ & 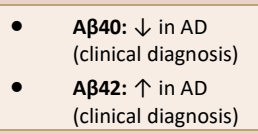 & $\begin{array}{l}P E T \\
A \beta 42: A U C=0.776\end{array}$ & $\begin{array}{l}P E T \\
A \beta 40:=-\mathbf{0 . 5 8 5} \\
A \beta 42: r=-0.281\end{array}$ & [24] \\
\hline & - $\quad$ Exosome-bound $A \beta 42$ & $\begin{array}{l}\text { Exosome-bound } \\
\text { A } 342: \uparrow \text { in A+ } \\
\text { group }\end{array}$ & & $\begin{array}{l}\text { PET } \\
\text { Exosome-bound } A \beta 42: r=0.9002\end{array}$ & [29] \\
\hline & $\begin{array}{l}\text { - A } \beta \text { - heterogeneous } \\
\text { (monomers + } \\
\text { oligomers/aggregates) } \\
\text { and monomerized } \\
\text { states }\end{array}$ & $\begin{array}{l}\text { - } \\
\text { ratio in } A D \\
\text { compared with } C U\end{array}$ & & $\begin{array}{l}\text { PET } \\
\text { Self -standard ratio (AMC): } r=0.5511 \\
\text { Self -standard ratio (KIRAMS): } r=0.4141\end{array}$ & [28] \\
\hline
\end{tabular}

Table 1. A summary of the results from studies investigating plasma amyloid as a biomarker of cerebral amyloidosis. 